\title{
РАСЧЕТ КОЭФФИЦИЕНТА СВЕРХСЖИМАЕМОСТИ ОСНОВНЫХ КОМПОНЕНТ ПРИРОДНОГО ГАЗА МЕТОДОМ МОЛЕКУЛЯРНОЙ ДИНАМИКИ
}

\author{
Малышев Виктор Леонидович', \\ Victor.L.malyshev@gmail.com \\ Моисеева Елена Флоридовна', \\ Elena.f.moiseeva@gmail.com \\ Калиновский Юрий Валентинович', \\ kafedrargkm@mail.ru
1 Уфимский государственный нефтяной технический университет, Россия, 450062, г. Уфа, ул. Космонавтов, 1.

\begin{abstract}
Актуальность работы обусловлена необходимостью определения точного значения коэффициента сверхсжимаемости для подсчета запасов газа, прогнозирования технологических показателей разработки газовых и газоконденсатных месторождений, поведения углеводородной системы при движении в лифтовых трубах, а также подготовки скважинной продукции и ее транспортировки.

Цель: разработка программного модуля расчета коэффициента сверхсжимаемости компонент природного газа для улучшения точности определения z-фактора в сравнении с классическим подходом, основанным на использовании уравнения состояния. Методы. Для определения z-фактора используется методика, основанная на использовании уравнения состояния Пенга-Робинсона, его модификация с шифт параметром, а также разработанный авторами программный модуль молекулярно-динамического моделирования, описывающий поведение системы на молекулярном уровне. В качестве модели межатомного взаимодействия частиц рассматривается потенциал Леннард-Джонса и NPT-ансамбль.

Результаты. На основе предложенных методов моделирования показано, что метод молекулярной динамики позволяет с достаточной точностью рассчитывать коэффициент сверхсжимаемости для основных углеводородных компонентов природных газов. На основе уравнения состояния Пенга-Робинсона и его модификации с шифт параметром, а также с помощью моделирования методом молекулярной динамики для метана, этана, пропана, азота и двуокиси углерода определены зависимости коэффициента сверхсжимаемости от давления в диапазоне температур от 250 до $410 \mathrm{~K}$. Произведено уточнение параметров потенциала Леннард-Джонса для этана, пропана, азота и двуокиси углерода, что позволило повысить точность при расчете коэффициента сверхсжимаемости. Показано, что средняя абсолютная ошибка метода молекулярной динамики практически во всех расчетах не превышает $3 \%$.
\end{abstract}

\section{Ключевые слова:}

Уравнение состояния, коэффициент сверхсжимаемости, моделирование, метод молекулярной динамики, потенциал Леннард-Джонса.

\section{Введение}

Описание и регулирование движения флюида в горной породе, скважинах и трубопроводе требует точных данных о свойствах нефти и газа в широком диапазоне давлений и температур.

Основным компонентом природных газов, представляющих интерес для газовой промышленности, является метан (60-95\%). Помимо метана природные газы содержат также более тяжелые углеводороды и неуглеводородные соединения, такие как диоксид углерода $\mathrm{CO}_{2}$, азот $\mathrm{N}_{2}$ и др. Количество тяжелых углеводородов в смеси, как правило, экспоненциально убывает с ростом числа атомов углерода в молекуле, однако наличие таких компонентов может оказывать существенное влияние на термодинамические свойства газа. Когда природный газ содержит значительную долю тяжелых компонентов (C5+), в пласте возможно проявление так называемых ретроградных явлений (образование жидкой фазы при изотермическом снижении давления в пласте). Выпадение конденсата при добыче газа является серьезной проблемой газодобывающей отрасли, в первую очередь, с экономической точки зрения. Кроме того, выпавший конденсат (жидкая фаза) затрудняет продвижение газа к забою скважины. Таким образом, точное описание фазового поведения углеводородов имеет особое значение при разработке газоконденсатных месторождений [1].

Одним из основных параметров, необходимых для прогнозирования фазовых переходов и расчетов количества газа, добываемого и транспортируемого под высоким давлением в широком диапазоне температур, является коэффициент сверхсжимаемости. В глубоко залегающих газовых пластах температура может достигать $200{ }^{\circ} \mathrm{C}$, а давление 120 МПа. Экспериментальные измерения поведения и термодинамических свойств в таких условиях сложны и требуют значительных финансовых затрат, поэтому необходимы альтернативные методы для получения надежных данных.

Основной подход к определению коэффициента сверхсжимаемости углеводородных смесей основан на использовании уравнения состояния. Несмотря на то, что большинство уравнений состояния было предложено еще в XX в., их сравнитель- 
ный анализ и модификация продолжается и по сей день $[2,3]$. Следует отметить, что практически все уравнения являются эмпирическими связями, которые требуют точной «настройки» коэффициентов парного взаимодействия между парами углеводородов, что не всегда возможно реализовать в условиях недостатка экспериментальных данных. Предсказание поведения газового конденсата по уравнениям состояния в промышленности, как правило, основано на использовании псевдокомпонентов, чьи термодинамические свойства усредняются для более точного описания фазового поведения. При таком подходе необходимо проводить серьезные исследования фазового и объемного поведения образцов газа, прежде чем проектировать разработку месторождения.

В данной работе предложен новый подход к расчету коэффициента сверхсжимаемости углеводородных компонентов природных газов. С помощью метода молекулярной динамики произведен расчет коэффициента сверхсжимаемости метана, этана, пропана, азота, двуокиси углерода в диапазоне давлений и температур, наиболее характерном для залежей углеводородов. Произведено сравнение результатов моделирования с результатами, полученными при использовании уравнения состояния Пенга-Робинсона и его модификации, а также с известными экспериментальными данными.

\section{Уравнение состояния Пенга-Робинсона и его модификация}

Использование уравнения состояния позволяет определить важнейшие теплофизические свойства вещества (сверхсжимаемость, теплоемкость, дроссель-эффект и т. д.), знание которых необходимо для рационального проектирования разработки газовых и газоконденсатных месторождений, а также в технологических процессах при транспортировке и переработке добываемого сырья.

В работе [2] приведен сравнительный анализ результатов применения уравнений состояния Coаве-Редлиха-Квонга, Пенга-Робинсона, PC-SAFT и SAFT-VR Mie для описания физических свойств компонент природного газа. На сегодняшний день в газовой промышленности есть попытки описания фазовых равновесий и термодинамических свойств с использованием уравнения состояния PC-SAFT. Однако в современных гидродинамических симуляторах в композиционных моделях, учитывающих фазовые переходы, используется уравнение состояния Пенга-Робинсона [4], получившее широчайшее распространение при моделировании фазового поведения углеводородов, которое и рассматривается в данной работе. Уравнение состояния Пенга-Робинсона (УС ПР) имеет вид:

$$
P=\frac{R T}{v-b}-\frac{a}{v(v+b)+b(v-b)},
$$

где $a, b$ - коэффициенты; $v$ - мольный объем; $R$ универсальная газовая постоянная.
Коэффициенты $a$ и $b$ для чистых веществ определяются только свойствами вещества и рассчитываются следующим образом:

$$
\begin{gathered}
a=a_{c} \varphi(T) ; \\
\varphi(T)=\left[1+\psi\left(1-\sqrt{T / T_{\text {кр }}}\right)\right]^{2} ; \\
\psi=0,37464+1,54226 \omega-0,26992 \omega^{2} ; \\
a_{c}=0,457235 R^{2} T_{\text {кр }}^{2} / P_{\text {кр }} ; \\
b=0,077796 R T_{\text {кр }} / P_{\text {кр }},
\end{gathered}
$$

где $P_{\text {кр }}, T_{\text {кр }}, \omega$ - соответственно критическое давление, критическая температура и ацентрический фактор чистого вещества.

Известна также модификация уравнения состояния Пенга-Робинсона, реализованная Б. Явери и Г. Юнгреном [5], в которой для мольного объема $v$ вводится поправка $c$ :

$$
\tilde{v}=v-c .
$$

Для расчета величины $c$ рекомендуют использовать так называемый «шифт-параметр» $s: c=s b$, приведенный в табл. 1.

Таблица 1. Шифт-параметр для компонент природного газа

Table 1. Shift-parameter for natural gas components

\begin{tabular}{|c|c|}
\hline Компонента/Component & $s$ \\
\hline $\mathrm{CH}_{4}$ & $-0,1595$ \\
\hline $\mathrm{C}_{2} \mathrm{H}_{6}$ & $-0,1134$ \\
\hline $\mathrm{C}_{3} \mathrm{H}_{8}$ & $-0,0863$ \\
\hline $\mathrm{N}_{2}$ & $-0,1927$ \\
\hline $\mathrm{CO}_{2}$ & $-0,0817$ \\
\hline
\end{tabular}

Для определения коэффициента сверхсжимаемости на основе уравнения состояния можно использовать обобщенное уравнение МенделееваКлапейрона для реального газа

$$
Z=\frac{P V}{R T} .
$$

Использование уравнения состояния Пенга-Робинсона позволяет определить коэффициент сверхсжимаемости на основе решения кубического уравнения:

$$
\begin{gathered}
Z^{3}-(1-B) Z^{2}+\left(A-3 B^{2}-2 B\right) Z- \\
-\left(A B-B^{2}-B^{3}\right)=0,
\end{gathered}
$$

где коэффициенты $A, B$ определяются по формулам:

$$
A=\frac{a P}{R^{2} T^{2}} ; B=\frac{b P}{R T} .
$$

\section{Метод молекулярной динамики}

Одним из современных подходов к исследованию фазового поведения углеводородных смесей является моделирование методом молекулярной динамики (МД). Данный подход позволяет напрямую рассчитывать динамику молекул системы, а также ее термодинамические свойства. 
Взаимодействие молекул определяется выбранным потенциалом взаимодействия. Для моделирование углеводородных систем широкое распространение получили потенциалы Ми и ЛеннардДжонса $[6,7]$. Наиболее известная и простая в реализации потенциальная функция для описания взаимодействия частиц - потенциал ЛеннардДжонса:

$$
U^{L J}\left(r_{i j}\right)=4 \varepsilon\left[\left(\frac{\sigma}{r_{i j}}\right)^{12}-\left(\frac{\sigma}{r_{i j}}\right)^{6}\right],
$$

где $r_{i j}=\left|r_{i}-r_{j}\right|-$ расстояние между молекулами $i$ и $j$, а $r_{i}, r_{j}$ - положения частиц $i$ и $j$ соответственно; $\varepsilon-$ глубина потенциальной ямы; $\sigma$ - расстояние, на котором энергия взаимодействия равна нулю.

Однако использование потенциала ЛеннардДжонса для углеводородов возможно лишь при замене молекулы углеводорода, состоящей из атомов двух веществ (углерода С и водорода Н), на одноатомную модель. В работе показано, что при соответствующих параметрах взаимодействия $\sigma$ и $\varepsilon$ потенциал Леннард-Джонса позволяет с достаточной точностью рассчитать коэффициент сверхсжимаемости компонент природного газа.

Для моделирования $z$-фактора компонентов природного газа методом молекулярной динамики будем рассматривать $N P T$-ансамбль, в котором количество частиц, давление и температура постоянны во времени. Детали метода молекулярной динамики и его реализации на гетерогенных вычислительных станциях можно найти в предыдущих работах авторов [8-10]. В качестве исходных данных задана кубическая область с числом молекул $N=8000$. Для сохранения температуры и давления в системе используется термостат Берендесена и процедура масштабирования Ньютона-Рафсона. Давление в системе рассчитывается путем осреднения мгновенной функции давления, полученной на основе вириального разложения:

$$
P=\frac{N k_{b} T}{V}+\left\langle\frac{1}{3 V} \sum_{i=1}^{N-1} \sum \vec{r}_{i j} \vec{f}_{i j}\right\rangle
$$

где $N, V$ и $T$ - число частиц, объем и температура соответственно; $k_{b}-$ константа Больцмана; $f_{i j}-$ сила, действующая на частицу $j$ со стороны частицы $i$.

В силу того, что моделирование производится при постоянных параметрах $N, P, T$, коэффициент сверхсжимаемости можно определить как отношение заданного давления в рассматриваемой системе к давлению, рассчитанному по уравнению состояния Менделеева-Клапейрона для идеального газа при соответствующей плотности вещества:

$$
Z_{M D}=\frac{P M}{\rho_{M D} R T},
$$

где $\rho_{M D}$ - плотность, соответствующая заданному давлению; $M$ - молярная масса; $R$ - универсальная газовая постоянная.

\section{Метан}

Экспериментальные исследования метана $\left(\mathrm{CH}_{4}\right)$ охватывают широкий температурный диапазон от 100 до $1000 \mathrm{~K}$, а область давлений от 0,1 до 100 МПа. В работе [11] приведено уравнение состояния метана, полученное на основе обобщения экспериментальных данных различных авторов. Оно позволяет с высокой точностью описывать PVT-свойства метана. При этом автором было отмечено, что погрешность в определении плотности во всем диапазоне экспериментальных данных не превышает $0,1 \%$, а для многих точек существенно меньше $(0,02-0,05 \%)$.

\begin{tabular}{|c|c|c|c|c|c|c|}
\hline $\mathrm{T}, \mathrm{K}$ & $\begin{array}{c}\mathrm{P}, \mathrm{M} \text { Ма } \\
\text { (MPa) }\end{array}$ & $\begin{array}{l}\rho, \mathrm{k} / \mathrm{M}^{3} \\
\left(\mathrm{~kg} / \mathrm{m}^{3}\right)\end{array}$ & $\begin{array}{l}Z \text { экс } \\
\text { (exp) }\end{array}$ & $Z_{\mathrm{IIP}} / Z_{\mathrm{PR}}$ & $\begin{array}{l}Z_{\Pi \mathrm{IP}+\text { shift }} \\
Z_{\mathrm{PR}+\text { shift }}\end{array}$ & $Z_{M D}$ \\
\hline \multirow{9}{*}{250} & 1 & 7,97 & 0,9682 & 0,9616 & 0,9636 & 0,9712 \\
\hline & 5 & 46,19 & 0,8355 & 0,8118 & 0,8217 & 0,8544 \\
\hline & 10 & 112,59 & 0,6855 & 0,6690 & 0,6889 & 0,6976 \\
\hline & 15 & 179,96 & 0,6433 & 0,6368 & 0,6666 & 0,6430 \\
\hline & 20 & 223,21 & 0,6915 & 0,6758 & 0,7154 & 0,7001 \\
\hline & 25 & 250,71 & 0,7696 & 0,7387 & 0,7883 & 0,7737 \\
\hline & 30 & 270,29 & 0,8566 & 0,8099 & 0,8693 & 0,8649 \\
\hline & 50 & 317,3 & 1,2162 & 1,1105 & 1,2096 & 1,2323 \\
\hline & 80 & 355,89 & 1,7349 & 1,5561 & 1,7147 & 1,7735 \\
\hline \multirow{9}{*}{300} & 1 & 6,54 & 0,9833 & 0,9978 & 0,9980 & 0,9831 \\
\hline & 5 & $\begin{array}{l}34,97 \\
\end{array}$ & 0,9196 & 0,9786 & 0,9803 & 0,9239 \\
\hline & 10 & 75,2 & 0,8553 & 0,9018 & 0,9100 & 0,8573 \\
\hline & 15 & 117,25 & 0,8228 & 0,8337 & 0,8502 & 0,8210 \\
\hline & 20 & 155,29 & 0,8283 & 0,8047 & 0,8295 & 0,8261 \\
\hline & 25 & $\begin{array}{ll}186,31 \\
\end{array}$ & 0,863 & 0,8107 & 0,8437 & 0,8584 \\
\hline & 30 & 210,82 & 0,9152 & 0,8398 & 0,8811 & 0,9168 \\
\hline & 50 & 271,62 & 1,1839 & 1,1007 & 1,1833 & 1,2027 \\
\hline & 80 & 319,62 & 1,6098 & 1,4575 & 1,5896 & 1,6222 \\
\hline \multirow{9}{*}{410} & 1 & 4,72 & 0,9961 & 0,9994 & 0,9995 & 0,9959 \\
\hline & 5 & $\begin{array}{l}23,92 \\
\end{array}$ & 0,9836 & 0,9938 & 0,995 & 0,9850 \\
\hline & 10 & 48,25 & 0,9753 & 0,9738 & 0,9798 & 0,9774 \\
\hline & 15 & 72,3 & 0,9763 & 0,96 & 0,9721 & 0,9783 \\
\hline & 20 & 95,38 & 0,9868 & 0,9578 & 0,9759 & 0,9892 \\
\hline & 25 & 116,95 & 1,006 & 0,9656 & 0,9897 & 1,0026 \\
\hline & 30 & 136,73 & 1,0326 & 0,9814 & 1,0116 & 1,0357 \\
\hline & 50 & 198,38 & 1,1861 & 1,1294 & 1,1898 & 1,1868 \\
\hline & 80 & 255,79 & 1,4718 & 1,3624 & 1,4591 & 1,4770 \\
\hline
\end{tabular}

Таблица 2. Значения коэффиииента сверхсжимаемости метана Table 2. Z-factor for methane

При разработке газовых и газоконденсатных месторождений полный цикл движения газожидкостной смеси включает в себя: фильтрацию в пористой среде, динамику смеси в лифтовых трубах, сепарацию, движение в трубопроводе. Все эти процессы сопровождаются постоянными изменениями температуры и давления. Учитывая климатические условия месторождений, существующий геотермический градиент, а также технологические условия процессов добычи углеводородов, основные свойства природных газов достаточно точно можно описать, рассмотрев область температур от 230 до 410 К. В табл. 2 приведены зависимости 
коэффициента сверхсжимаемости метана $Z$ от давления $P$ для различных значений температур. Анализ результатов показывает, что наилучшее совпадение с экспериментальными данными получено при расчете $Z$ методом молекулярной динамики $\left(Z_{M D}\right)$, а также при использовании уравнения состояния Пенга-Робинсона с шифт-параметром $\left(Z_{\Pi \text { IР+shift }}\right)$. Из данных таблицы видно, что в области высоких давлений уравнение Пенга-Робинсона $\left(Z_{\text {пр }}\right)$ дает значительную ошибку $(>10$ \%). Максимальная относительная ошибка для метода МД достигается при $250 \mathrm{~K}$ и составляет $2,27 \%$, в то время как для УС ПР с шифт-параметром - 3,62\%. Кроме того, при использовании метода МД и УС ПР с шифт-параметром нельзя выделить систематической зависимости величины относительной ошибки от давления.

\section{Этан}

Экспериментальные данные о термодинамических свойствах этана $\left(\mathrm{C}_{2} \mathrm{H}_{6}\right)$ представлены в области температур от тройной точки до $700 \mathrm{~K}$ и давлений от 0,1 до 80 МПа [12].

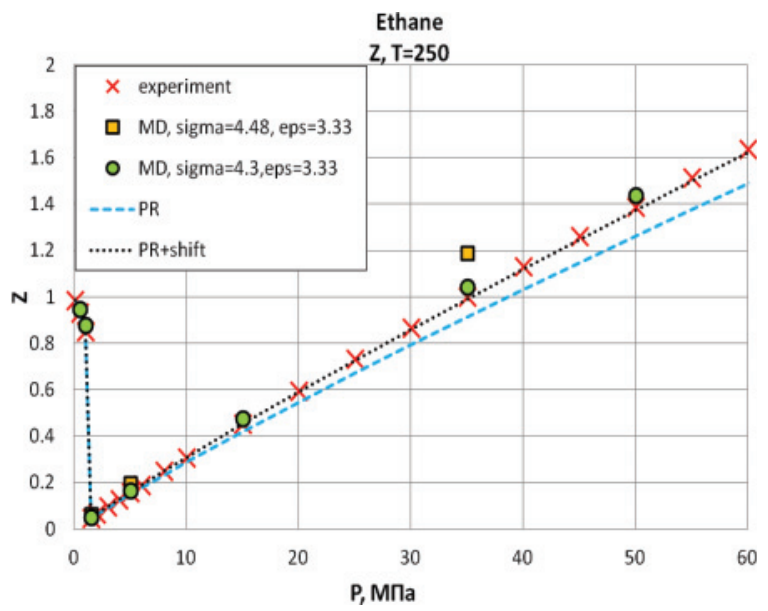

Pис. 1. Коэффициент сверхсжимаемости этана при телпераmyре $\mathrm{T}=250 \mathrm{~K}$

Fig. 1. Compressibility factor for ethane at $T=250 \mathrm{~K}$

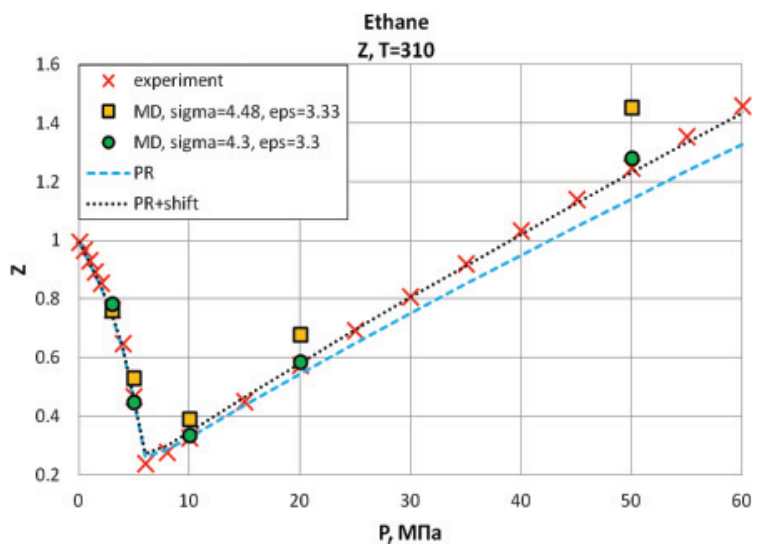

Pис.2. Коэффициент сверхсжимаемости этана при телпераmype $\mathrm{T}=300 \mathrm{~K}$

Fig. 2. Compressibility factor for ethane at $T=300 \mathrm{~K}$

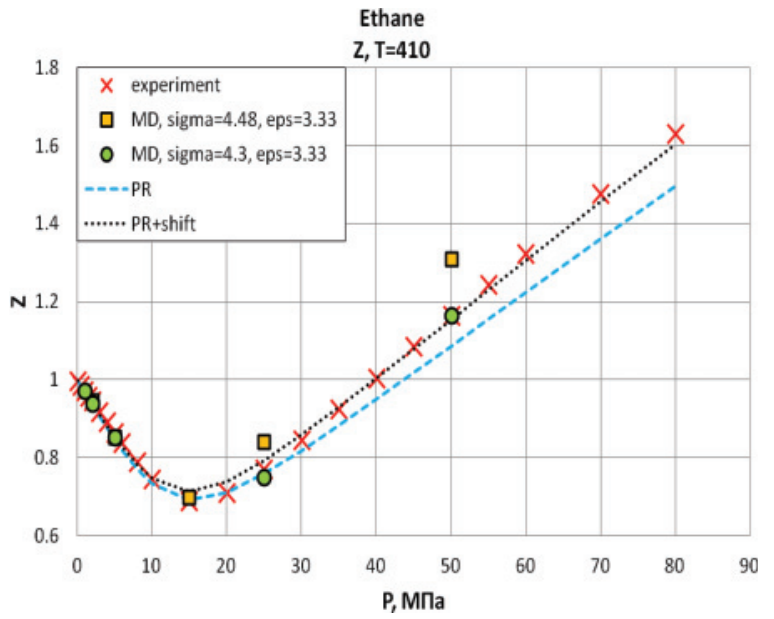

Pис. 3. Коэффициент сверхсжилаемости этана при телпера mype $T=410 \mathrm{~K}$

Fig. 3. Compressibility factor for ethane at $\mathrm{T}=410 \mathrm{~K}$

В данной работе нами был рассчитан коэффициент сверхсжимаемости этана в диапазоне температур от 250 до 410 К. На рис. 1-3 представлены результаты, рассчитанные методом МД, по УС Пенга-Робинсона, а также данные, полученные согласно УС ПР с шифт-параметром. Стоит отметить, что расчет при параметрах Леннарда-Джонса из [13], равных $\sigma=4,48 \AA, \varepsilon=3,33 \cdot 10^{-21}$ Дж дал неудовлетворительный результат, что говорит о невозможности использования данных параметров для определения коэффициента Z. Одно из направлений, которое широко используется в физико-химической области, - это адаптация параметров потенциала для описания свойств веществ и их смесей [6]. Поэтому в представленной работе были предложены корректировки параметров потенциала и было обнаружено, что использование параметра $\sigma=4,3 \AA$ позволяет значительно снизить относительную ошибку в расчете коэффициента сверхсжимаемости. Если в первом случае максимальная точечная погрешность достигала $27 \%$, то при $\sigma=4,3 \AA$ максимальная ошибка наблюдалась при $T=250$ К и $P=1,5$ МПа и составила $6 \%$. При этом стоит отметить, что УС ПР также давало значительную ошибку в определении коэффициента сверхсжимаемости этана, но использование УС ПР с шифт-параметром позволило значительно увеличить точность расчетов. Из графиков видно, что при температуре $T=250 \mathrm{~K}$ наилучшее совпадение с экспериментальными данными достигается при использовании УС ПР с шифт-параметром, однако при более высоких температурах точность расчета коэффициента сверхсжимаемости методом МД оказывается выше.

\section{Пропан}

Пропан $\left(\mathrm{C}_{3} \mathrm{H}_{8}\right)$ - еще один компонент природного газа, который часто используется в качестве топлива в быту. Сложность при определении термодинамических свойств пропана расчетными методами заключается в том, что для него характерна 
чрезвычайно широкая по температурам область существования жидкости. В таких условиях аппроксимация термодинамических свойств единым уравнением состояния, когда свойства жидкости определяются непрерывным интегрированием уравнения на изотерме от идеального газового до жидкого с переходом через двухфазную области, становится весьма затруднительной. Поэтому прямое моделирование пропана методом молекулярной динамики может стать хорошей альтернативой экспериментальным и теоретическим методам исследования.

На рис. 4-6 приведены результаты расчетов коэффициента сверхсжимаемости пропана с помощью моделирования методом молекулярной динамики, с использованием УС ПР и УС ПР с шифтпараметром, а также экспериментальные данные [14]. Из графиков видно, что УС ПР дает существенную погрешность при расчете коэффициента $\mathrm{Z}$ в области высоких давлений. Эту особенность можно обойти, используя УС ПР с шифт-параметром. При этом можно видеть, что метод молекулярной динамики также показывает хорошие результаты, как в области низких, так и в области высоких давлений. Стоит отметить, что параметры потенциала взаимодействия Леннард-Джонса $\sigma$ и $\varepsilon$, приведенные в [13], показали неудовлетворительные результаты при расчете коэффициента сверхсжимаемости, поэтому нами была произведена работа по подбору наилучших параметров. Таким образом, наибольшую точность при расчете $Z$ показали значения $\sigma=4,77 \AA$, $\varepsilon=4,28 \cdot 10^{-21}$ Дж.

\section{Азот и двуокись углерода}

Азот - инертный газ, который является частой примесью в составе углеводородного газа. Его содержание в природных газах незначительно, но на некоторых месторождениях существуют значительные отложения азотного газа.

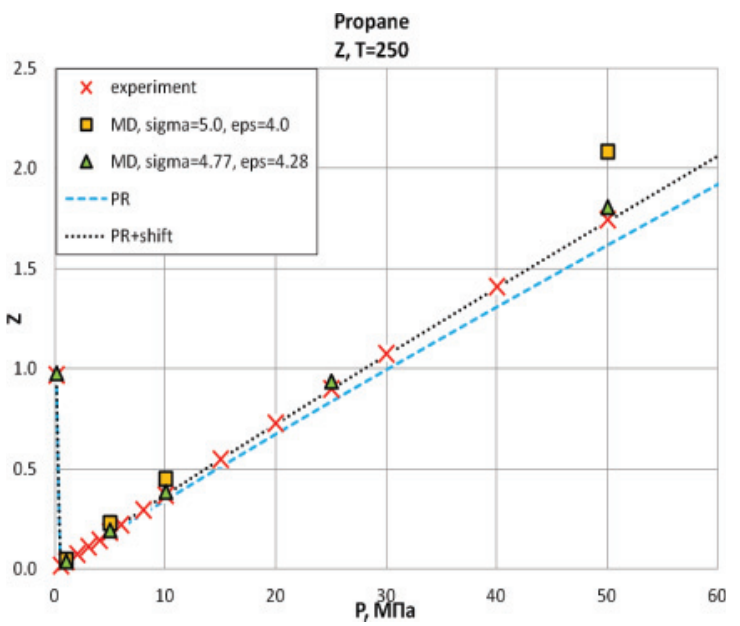

Pис. 4. Коэффициент сверхсжимаемости пропана при телпера mype $\mathrm{T}=250 \mathrm{~K}$

Fig. 4. Compressibility factor for propane at $T=250 \mathrm{~K}$

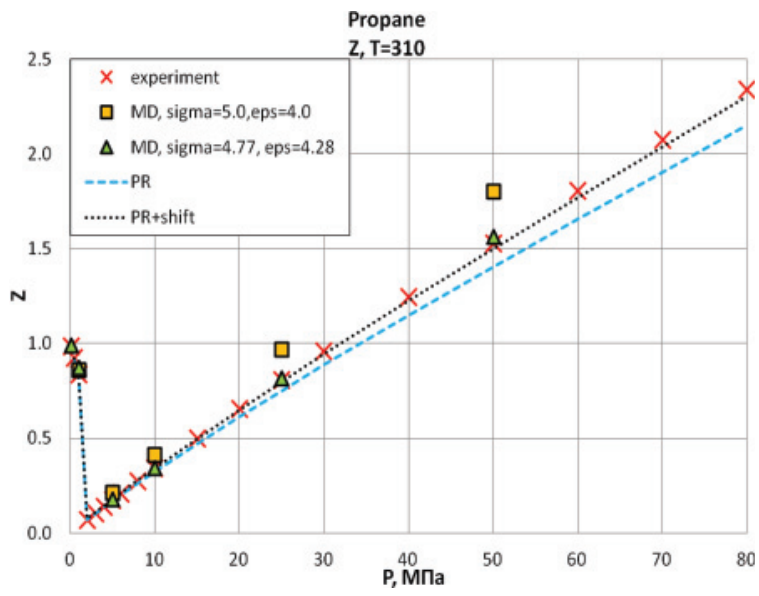

Рис. 5. Коэффициент сверхсжимаемости пропана при телпера mype $T=310 \mathrm{~K}$

Fig. 5. Compressibility factor for propane at $T=310 \mathrm{~K}$

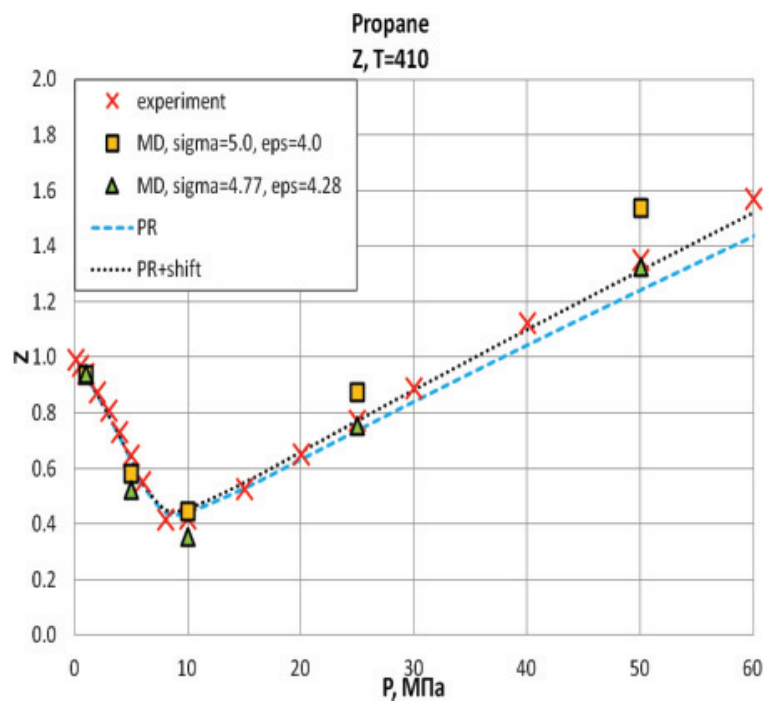

Pис. 6. Коэффициент сверхсжимаемости пропана при телпера mype $\mathrm{T}=410 \mathrm{~K}$

Fig. 6. Compressibility factor for propane at $T=410 \mathrm{~K}$

Двуокись углерода и сероводород появляются в газовой смеси за счет окисления углеводородов при помощи кислорода и наличия аэробных бактерий. При соприкосновении углеводородов с сульфатными пластовыми водами образуется как углекислый газ, так и сероводород. Таким образом, сероводород, сера и углекислый газ практически постоянно присутствуют в составе углеводородного газа.

Газы практически всех месторождений содержат двуокись углерода и азота, которые существенно влияют на сжимаемость газовой смеси. Поэтому аналогичным образом для азота и углекислого газа проведены расчеты коэффициента сверхсжимаемости по уравнению состояния Пенга-Робинсона, его модификации с шифт-параметром и методом молекулярной динамики в сравнении с экспериментальными данными $[15,16]$. Моделирование динамики молекул двуокиси углеро- 
да как полярной жидкости требует учета взаимодействия всех пар атомов: С-С, О-О, С-О. Однако в работе показано, что, пренебрегая атомными взаимодействиями и учитывая лишь межмолекулярные взаимодействия центров масс, можно получить удовлетворительные результаты.

Анализ литературы [17-20] показывает, что параметры потенциала Леннард-Джонса для компонент природного газа разнятся в различных статьях в зависимости от области исследования авторов. В табл. 3 авторами предложен набор параметров $\sigma, \varepsilon$, которые позволяют с достаточной точностью определять коэффициент сверхсжимаемости в области давлений и температур, характерных для полного цикла транспортировки и разработки газовых и газоконденсатных месторождений.

Таблица 3. Адаптированные паралетры потенциала ЛеннардДжонса

Table 3. Modified parameters for the Lennard-Jones

\begin{tabular}{|c|c|c|c|c|c|}
\hline $\begin{array}{c}\text { Параметры потенциала } \\
\text { Potential parameters }\end{array}$ & $\mathrm{CH}_{4}$ & $\mathrm{C}_{2} \mathrm{H}_{6}$ & $\mathrm{C}_{3} \mathrm{H}_{8}$ & $\mathrm{~N}_{2}$ & $\mathrm{CO}_{2}$ \\
\hline$\sigma, \AA$ & 3,73 & 4,3 & 4,77 & 3,625 & 3,72 \\
\hline$\varepsilon \cdot 10^{-21}$ Дж (J) & 2,042 & 3,33 & 4,28 & 1,32 & 3,258 \\
\hline
\end{tabular}

Средняя абсолютная ошибка, рассчитанная по формуле $\Delta=\frac{100}{N} \sum_{i=1}^{N}\left(Z_{i}^{\text {exp }}-Z_{i}^{\text {method }}\right)$, для предложенных методов с использованием вышеупомянутых параметров потенциала Леннард-Джонса приведена в табл. 4.

Анализ результатов показывает, что практически для всех компонент наибольшей точностью определения коэффициента сверхсжимаемости во всем интервале исследуемых температур обладает метод молекулярной динамики и уравнение состояния Пенга-Робинсона с шифт-параметром. Однако шифт-параметр ухудшает результаты определения термодинамических свойств, таких как теплоемкость и дифференциальный коэффициент Джоуля-Томсона по сравнению с классическим уравнением Пенга-Робинсона, что показано в работе [21]. Таким образом, метод молекулярной динамики может стать хорошим альтернативным методом определения термодинамических свойств компонент природного газа и их смесей.

\section{СПИСОК ЛИТЕРАТУРЫ}

1. Анализ причин роста газового фактора на поздних стадиях разработки нефтяных месторождений / М.К. Баймухаметов, Д.С. Гулишов, В.Г. Михайлов, А.И. Пономарев // Известия Томского политехнического университета. Инжиниринг георесурсов. - 2018. - Т. 329. - № 8. - С. 104-111

2. Comparative study of vapour-liquid equilibrium and density modelling of mixtures related to carbon capture and storage with the SRK, PR, PC-SAFT and SAFT-VR Mie equations of state for industrial uses / A.G. Perez, C. Coquelet, P. Paricaud, A. Chapoy // Fluid Phase Equilibria. - 2017. - V. 440 - P. 19-35. таблица 4. Средняя абсолютная ошибка рассматриваемых методов для различных телператур

Table 4. Average absolute error of the considered methods at different temperatures

\begin{tabular}{|c|c|c|c|}
\hline \multirow[b]{2}{*}{$\begin{array}{l}\text { Tемпература, } \mathrm{K} \\
\text { Temperature, } \mathrm{K}\end{array}$} & \multicolumn{3}{|c|}{$\begin{array}{l}\text { Средняя абсолютная ошибка, \% } \\
\text { Average absolute relative error, \% }\end{array}$} \\
\hline & $\begin{array}{l}\text { УС ПР/EOS PR } \\
\text { (Equation of state } \\
\text { Peng-Robinson) }\end{array}$ & $\begin{array}{l}\text { УС ПР-шифт } \\
\text { EOS PR-shift }\end{array}$ & $\begin{array}{c}\text { МД/MD } \\
\text { (molecular } \\
\text { dynamics) }\end{array}$ \\
\hline \multicolumn{4}{|c|}{$\mathrm{CH}_{4}$} \\
\hline 250 & 4,79 & 1,41 & 1,22 \\
\hline 300 & 5,75 & 2,59 & 0,53 \\
\hline 410 & 3,53 & 0,98 & 0,23 \\
\hline \multicolumn{4}{|c|}{$\mathrm{C}_{2} \mathrm{H}_{6}$} \\
\hline 250 & 3,75 & 0,43 & 2,46 \\
\hline 310 & 4,04 & 1,21 & 2,02 \\
\hline 410 & 2,39 & 1,03 & 0,80 \\
\hline \multicolumn{4}{|c|}{$\mathrm{C}_{3} \mathrm{H}_{8}$} \\
\hline 250 & 3,89 & 0,37 & 2,52 \\
\hline 310 & 3,63 & 0,83 & 1,41 \\
\hline 410 & 3,26 & 1,57 & 3,87 \\
\hline \multicolumn{4}{|c|}{$\mathrm{N}_{2}$} \\
\hline 250 & 5.91 & 0.73 & 0.40 \\
\hline 300 & 5.41 & 0.57 & 0.43 \\
\hline 400 & 4.13 & 0.37 & 0.40 \\
\hline \multicolumn{4}{|c|}{$\mathrm{CO}_{2}$} \\
\hline 250 & 3.67 & 0.21 & 4.98 \\
\hline 300 & 2.44 & 1.19 & 3.40 \\
\hline 400 & 2.28 & 1.88 & 1.30 \\
\hline
\end{tabular}

\section{Заключение}

В работе показано, что метод молекулярной динамики позволяет с достаточной точностью рассчитывать коэффициент сверхсжимаемости для основных углеводородных компонентов природных газов. На основе уравнения состояния Пенга-Робинсона и его модификации с шифт-параметром, а также с помощью моделирования методом молекулярной динамики для метана, этана, пропана, азота и двуокиси углерода определены зависимости коэффициента сверхсжимаемости от давления в диапазоне температур 250-410 К. Произведено уточнение параметров потенциала Леннард-Джонса для этана, пропана, азота и двуокиси углерода, что позволило повысить точность при расчете коэффициента сверхсжимаемости.

Работа выполнена при поддержке Российского научного фонда (грант РНФ 17-79-10094).

3. A comparison of PC-SAFT and PR-Peneloux equations of state in predicting the compressibility factors of lean gas-carbon dioxide mixtures at high pressures / M. Ebrahimi, S.A. Mousavi-Dehghani, B. Dabir A. , Shahrabadi // Journal of Natural Gas Science and Engineering. - 2016. - V. 31. - P. 681-691.

4. Peng D.Y., Robinson D.B. A new two-constant equation of state // Industrial \& Engineering Chemistry Fundamentals. - 1976. V. 15. - P. 59-64.

5. Jhavery B.S., Youngren G.K. Three-parameter modification of the Peng-Robinson equation of state to improve volumetric predictions // SPE Reservoir Engineering. - 1988. - V. 3. - № 3. P. 1033-1040. 
6. Developing Intermolecular-Potential Models for Use with the SAFT-VR Mie Equation of State / S. Dufal, T. Lafitte, A. Galindo, G. Jackson, A.J. Haslam // American Institute of Chemical Engineers Journal. - 2015. - V. 61. - № 9. - P. 2891-2912.

7. Магомедов М.Н. О вычислении параметров потенциала Ми-Леннарда-Джонса // Теплофизика высоких температур. 2006. - T. 44. - № 4. - C. 518-533.

8. Исследование прочности жидкости на разрыв методами молекулярной динамики / В.Л. Малышев, Д.Ф. Марьин, Е.Ф. Моисеева, Н.А. Гумеров, И.Ш. Ахатов // Теплофизика высоких температур. - 2015. - Т. 53. - № 3. - С. 423-429.

9. Влияние газа на прочность жидкости на разрыв. Моделирование методами молекулярной динамики / В.Л. Малышев, Д.Ф. Марьин, Е.Ф. Моисеева, Н.А. Гумеров // Теплофизика высоких температур. - 2016. - Т. 54. - № 4. - С. 640-644.

10. FMM/GPU accelerated molecular dynamics simulation of phase transitions in water-nitrogen-metal systems / E. Moiseeva, C. Mikhaylenko, V. Malyshev, D. Maryin, N. Gumerov // ASME 2012 International Mechanical Engineering Congress and Exposition, IMECE 2012. - Houston, Texas, 2012. - P. 883-892.

11. Сычев В.В., Вассерман А.А., Загорученко В.Л., Козлов А.Д., Спиридонов Г.А., Цымарный В.А. Термодинамические свойства метана. - Москва: Издательство стандартов, 1979. - 348 с.

12. Сычев В.В., Вассерман А.А., Загорученко В.Л., Козлов А.Д., Спиридонов Г.А., Цымарный В.А. Термодинамические свойства этана. - Москва: Издательство стандартов, 1982. - 304 с.

13. Yoshida T., Uematsu M. Prediction of PVT properties of natural gases by molecular simulation // Transactions of the Japan Society of Mechanical Engineers. Series B. - 1996. - V. 593. № 62. - P. 278-283.
14. Термодинамические свойства пропана / В.В. Сычев, А.А. Вассерман, А.Д. Козлов, В.А. Цымарный. - М.: Изд-во стандартов, 1989. - 268 с.

15. Термодинамические свойства азота / В.В. Сычев, А.А. Вассерман, А.Д. Козлов, Г.А. Спиридонов, В.А. Цымарный. - М.: Изд-во стандартов, 1977. - 352 с.

16. Алтунин В.В. Теплофизические свойства двуокиси углерода. М.: Изд-во стандартов, 1975. - 546 с.

17. Errington J.R., Panagiotopoulos A.Z. A new intermolecular potential model for the n-Alkane homologous series // Journal of Physical Chemistry B. - 1999. - V. 103. - P. 6314-6322.

18. Heyes D.M. Molecular dynamics simulations of liquid binary mixtures: Partial properties of mixing and transport coefficients // The Journal of Chemical Physics. - 1992. - V. 96. - № 3. P. 2217-2227.

19. Prediction of thermodynamic derivative properties of Cuids by Monte Carlo simulation / M. Lagache, P. Ungerer, A. Boutina, A.H. Fuchs // Physical Chemistry Chemical Physics. - 2001. V. 3. - P. 4333-4339.

20. Tchouar N., Benyettou M., Kadour F.0. Thermodynamic, structural and transport properties of Lennard-Jones liquid systems. A molecular dynamics simulations of liquid helium, neon, methane and nitrogen // International Journal of molecular sciences. - 2003. - № 4. - P. 595-606.

21. Малышев В.Л., Моисеева Е.Ф., Калиновский Ю.В. Сравнительный анализ определения термодинамических свойств метана на основе уравнения состояния Пенга-Робинсона и метода молекулярной динамики // Научные труды НИПИ Нефтегаза ГHKAP. - 2018. - № 2. - C. 33-40.

Поступила 14.02.2019 2.

\section{Информация об авторах}

Малышев В.Л., кандидат физико-математических наук, доцент кафедры разработки и эксплуатации газовых и нефтегазоконденсатных месторождений Уфимского государственного нефтяного технического университета.

Moисеева E.Ф., кандидат физико-математических наук, доцент кафедры разработки и эксплуатации газовых и нефтегазоконденсатных месторождений Уфимского государственного нефтяного технического университета.

Калиновский Ю.В., кандидат технических наук, доцент кафедры разработки и эксплуатации газовых и нефтегазоконденсатных месторождений Уфимского государственного нефтяного технического университета. 
UDC 533.21

\title{
CALCULATION OF COMPRESSIBILITY FACTOR OF MAIN NATURAL GAS COMPONENTS BY MEANS OF MOLECULAR DYNAMICS SIMULATIONS
}

\author{
Victor L. Malyshev', \\ Victor.L.malyshev@gmail.com \\ Elena F. Moiseeva', \\ Elena.f.moiseeva@gmail.com \\ Yurii V. Kalinovsky',
kafedrargkm@mail.ru
'Ufa State Petroleum Technological University,
1, Kosmonavtov street, Ufa, 450062, Russia.
}

The relevance of the work is caused by the need to determine the exact value of the compressibility factor for calculating gas reserves, forecasting technological indicators for development of gas and gas condensate fields, the behavior of hydrocarbon systems flow in wells, and preparation of well production and its transportation.

The aim of the research is to develop a program for computation of the compressibility factor for the main components of natural gas to achieve more accuracy in determining z-factor in comparison with classical approach, based on the equations of state.

Methods. To determine the z-factor the authors have used the technique based on the Peng-Robinson equation of state, its modification with a shift parameter, and a software module for molecular dynamic simulation developed by the authors that describes the behavior of the system at the molecular level. The potential of Lennard-Jones and NPT-ensemble is considered as a model of interatomic interaction of particles.

Results. Based on the proposed methods of modeling, it was shown that the molecular dynamics simulations make it possible to accurately calculate the compressibility factor for the main components of natural gases. Based of the Peng-Robinson equation of state and its modification with a shift parameter, as well as using the molecular dynamics simulation for methane, ethane, propane, nitrogen and carbon dioxide, the dependencies of the compressibility factor on pressure were determined in the temperature range from 250 to $410 \mathrm{~K}$. Lennard-Jones potential parameters for ethane, propane, nitrogen and carbon dioxide were specified that made it possible to improve the accuracy in calculating the compressibility factor. It is shown that the average absolute error of the molecular dynamics method in almost all calculations does not exceed $3 \%$.

Key words:

Equation of state, compressibility factor, simulations, molecular dynamics method, Lennard-Jones potential.

The research was supported by the Russian Science Fund (grant RSF 17-79-10094).

\section{REFERENCES}

1. Baimukhametov M.K., Gulishov D.S., Mikhaylov V.G., Ponomarev A.I., Topolnikov A.S. Analysis of causes of gas-oil ratio growth at late stages of oil fields exploration. Bulletin of the Tomsk Polytechnic University. Geo Assets Engineering, 2018, vol. 329, no. 8, pp. 104-111. In Rus.

2. Perez A.G., Coquelet C., Paricaud P., Chapoy A. Comparative study of vapour-liquid equilibrium and density modelling of mixtures related to carbon capture and storage with the SRK, PR, PC-SAFT and SAFT-VR Mie equations of state for industrial uses. Fluid Phase Equilibria, 2017, vol. 440, pp. 19-35.

3. Ebrahimi M., Mousavi-Dehghani S.A., Dabir B., Shahrabadi A. A comparison of PC-SAFT and PR-Peneloux equations of state in predicting the compressibility factors of lean gas-carbon dioxide mixtures at high pressures. Journal of Natural Gas Science and Engineering, 2016, vol. 31, pp. 681-691.

4. Peng D.Y., Robinson D.B. A new two-constant equation of state. Industrial \& Engineering Chemistry Fundamentals, 1976, vol. 15 , pp. 59-64.

5. Jhavery B.S., Youngren G.K. Three-parameter modification of the Peng-Robinson equation of state to improve volumetric predictions. SPE Reservoir Engineering, 1988, vol. 3, no. 3, pp. 1033-1040.

6. Dufal S., Lafitte T., Galindo A., Jackson G., Haslam A.J. Developing intermolecular-potential models for use with the SAFT-VR
Mie equation of state. American Institute of Chemical Engineers Journal, 2015, vol. 61, no. 9, pp. 2891-2912.

7. Magomedov M.N. The calculation of the parameters of the MieLennard-Jones potential. High Temperature, 2006, vol. 44, no. 4, pp. $513-529$.

8. Malyshev V.L., Marin D.F., Moiseeva E.F., Gumerov N.A., Akhatov I.S. Study of the tensile strength of a liquid by molecular dynamics methods. High Temperature, 2015, vol. 53, no 3, pp. 406-412.

9. Malyshev V.L., Moiseeva E.F., Marin D.F., Gumerov N.A. Influence of gas on the rupture strength of liquid: simulation by the molecular dynamics methods. High Temperature, 2016, vol. 54, no. 4, pp. 607-611.

10. Moiseeva E., Mikhaylenko C., Malyshev V., Maryin D., Gumerov N. FMM/GPU accelerated molecular dynamics simulation of phase transitions in water-nitrogen-metal systems. ASME 2012 International Mechanical Engineering Congress and Exposition, IMECE 2012. Houston, Texas, 2012. pp. 883-892.

11. Sychev V.V., Vasserman A.A., Zagoruchenko V.L., Kozlov A.D., Spiridonov G.A., Tsymarny V.A. Termodinamicheskie svoystva metana [Thermodynamic properties of methane]. Moscow, Standarts Publ., 1979. 348 p.

12. Sychev V.V., Vasserman A.A., Zagoruchenko V.L., Kozlov A.D., Spiridonov G.A., Tsymarny V.A. Termodinamicheskie svoystua etana [Thermodynamic properties of ethane]. Moscow, Standarts Publ., 1982. 304 p. 
13. Yoshida T., Uematsu M. Prediction of PVT properties of natural gases by molesular simulation. Transactions of the Japan Society of Mechanical Engineers. Series B, 1996, vol. 593, no. 62, pp. 278-283.

14. Sychev V.V., Vasserman A.A., Kozlov A.D., Tsymarny V.A. Termodinamicheskie svoystva propana [Thermodynamic properties of propane]. Moscow, Standarts Publ., 1989. 268 p.

15. Sychev V.V., Vasserman A.A., Kozlov A.D., Spiridonov G.A., Tsymarny V.A. Termodinamicheskie svoystva azota [Thermodynamic properties of nitrogen]. Moscow, Standarts Publ., 1977. $352 \mathrm{p}$.

16. Altunin V.V. Teplofizicheskie svoystva dvuokisi ugleroda [Thermophysical properties of carbon dioxide]. Moscow, Standarts Publ., 1975. $546 \mathrm{p}$.

17. Errington J.R., Panagiotopoulos A.Z. A new intermolecular potential model for the n-Alkane homologous series. Journal of Physical Chemistry B, 1999, vol. 103, pp. 6314-6322.

18. Heyes D.M. Molecular dynamics simulations of liquid binary mixtures: Partial properties of mixing and transport coefficients.

\section{Information about the authors}

Victor L. Malyshev, Cand. Sc., associate professor, Ufa State Petroleum Technological University.

Elena F. Moiseeva, Cand. Sc., associate professor, Ufa State Petroleum Technological University.

Yurii V. Kalinovsky, Cand. Sc., associate professor, Ufa State Petroleum Technological University.
The Journal of Chemical Physics, 1992, vol. 96, no. 3, pp. 2217-2227.

19. Lagache M., Ungerer P., Boutina A., Fuchs A.H. Prediction of thermodynamic derivative properties of Cuids by Monte Carlo simulation. Physical Chemistry Chemical Physics, 2001, vol. 3, pp. $4333-4339$.

20. Tchouar N., Benyettou M., Kadour F.0. Thermodynamic, structural and transport properties of Lennard-Jones liquid systems. A molecular dynamics simulations of liquid helium, neon, methane and nitrogen. International Journal of molecular sciences, 2003, no. 4, pp. 595-606.

21. Malyshev V.L., Moiseeva E.F., Kalinovsky Yu.V. Comparative study of the determination of thermodynamic properties of methane based on the peng-robinson equation of state and the molecular dynamics simulation. Socar Proceedings, 2018, no. 2, pp. 33-40. In Rus.

Received: 14 February 2019. 